Reprod. Nutr. Dévelop., 1988, 28 (1), 193-194.

\title{
Apolipoprotéines des lipoprotéines de la vache laitière
}

Maryse AYRAULT-JARRIER, Jacqueline BURDIN, Ginette THOMAS, A. MAZUR (*), Y. RAYSSIGUIER (*)

U.A. C.N.R.S. 524, Laboratoire de Biochimie,

Faculté de Médecine Saint-Antoine, 75571 Paris Cedex 12.

(*) Laboratoire des Maladies Métaboliques,

I.N.R.A., Theix, 63122 Ceyrat.

Summary. Plasma lipoproteins in cows were separated by ultracentrifugal flotation during the gestation-lactation cycle. It is concluded that apo A-I was the major apolipoprotein in all lipoprotein fractions. The concentrations of other apolipoproteins (apo $\mathrm{E}, \mathrm{C}, \mathrm{A}-\mathrm{IV}$ ) in $\mathrm{LDL}, \mathrm{HDL}_{2}$ and $\mathrm{HDL}_{3}$ were dependent on the physiologic status of the dairy cows.

De précédents travaux ont permis de déterminer la composition en lipides des différentes classes de lipoprotéines du sérum de la vache laitière (Stead et Welch, 1975 ; Gloeckler, Ferreri et Flaim, 1980 ; Grummer et Davis, 1984) et I'évolution de celles-ci au cours de différents états physiologiques de ces animaux (Raphael, Dimick et Puppione, 1973 ; Mazur et al., 1986). L'apparition de deux lipoprotéines de mobilité en agarose différente dans la zone des LDL (Puppione et al., 1972) de la vache lactante et gestante rendait nécessaire la détermination des apolipoprotéines de chaque classe de lipoprotéines. La caractérisation de ces apolipoprotéines et leur évolution au cours du cycle de gestation et de lactation de la vache a fait l'objet de ce travail.

Matériel et méthodes. Trois périodes caractéristiques du cycle de gestation et de lactation de 4 vaches laitières Pies Noires, issues d'un même troupeau et nourries à l'ensilage de maïs ont été étudiées. Les prélèvements sanguins ont été effectués, à la veine jugulaire, le matin après distribution des aliments, avant vêlage au cours de la période de tarissement (T), en début de lactation 7 à 10 jours après vêlage (V) et en période de pleine lactation 6 semaines après vêlage (L). Les lipoprotéines ont été séparées par ultracentrifugation séquentielle : après avoir fait flotter les VLDL densité < 1,006, l'augmentation à 1,060 de la densité du sérum débarrassé des VLDL a permis l'isolement des LDL $(1,006<d<1,060)$. Les lipoprotéines HDL ont été séparées en deux sousclasses $\left(\mathrm{HDL}_{2} 1,060<\mathrm{d}<1,125\right.$ et $\left.\mathrm{HDL}_{3} 1,125<\mathrm{d}<1,210\right)$. Les apolipoprotéines de chaque classe de densité ont été caractérisées suivant leurs masses molaires déterminées à l'aide de marqueurs de MM connues après électrophorèse en gradient $3-20 \%$ de gel de polyacrylamide en présence de $0,1 \%$ de SDS (Dodecyl-sulfate de sodium).

Résultats et discussion. Les lipoprotéines passent par un minimum dans la période $V(0,7 \mathrm{~g} / \mathrm{L}$ d'apolipoprotéines) et par un maximum dans la période $\mathrm{L}$ $(1,5 \mathrm{~g} / \mathrm{L})$. Contrairement aux lipoprotéines humaines, les $\mathrm{HDL}$ sont les lipoprotéines les plus importantes chez les ruminants, représentant $75 \%$ de l'ensemble des lipoprotéines circulantes. Les VLDL sont en trop faible quantité pour déterminer leur contenu en apolipoprotéines. Les apolipoprotéines des LDL représentent $14 \%, 8 \%, 13 \%$, des apolipoprotéines totales plasmatiques dans les périodes $T, V, L$, respectivement. L'apolipoprotéine majeure circulante chez ces animaux est une apolipoprotéine de MM 28 KD similaire à l'apo A-I humaine. Elle est présente dans toutes les lipoprotéines, quelle que soit leur densité. Mais si elle est presque la seule apolipoprotéine des $\mathrm{HDL}_{2}$, elle est accompagnée dans les $\mathrm{HDL}_{3}$ d'apolipoprotéines $\mathrm{C}$ de MM $12 \mathrm{KD}$ et de deux apolipoprotéines de $\mathrm{MM}$ 
environ $35 \mathrm{KD}$ et $46 \mathrm{KD}$ correspondant aux MM de l'apo $\mathrm{E}$ et de l'apo A-IV humaine respectivement. La baisse de concentration des apo $C$, E et A-IV dans les $\mathrm{HDL}_{3}$ est concomitante à leur augmentation dans les LDL ainsi qu'à celle de l'apo $B$, chez la vache en pleine lactation (fig. 1). En conclusion, malgré une forte concentration d'apo A-I dans les HDL de la vache laitière, I'existence d'autres apolipoprotéines (E et A-IV principalement) a pu être démontrée. La présence de ces apolipoprotéines, dans la zone de densité 1,006 - 1,060 pendant la période de pleine lactation, suggère un déplacement de particules de la zone $1,060-1,21$ vers une zone de densité plus faible.

\section{ELECTROPHORESE}

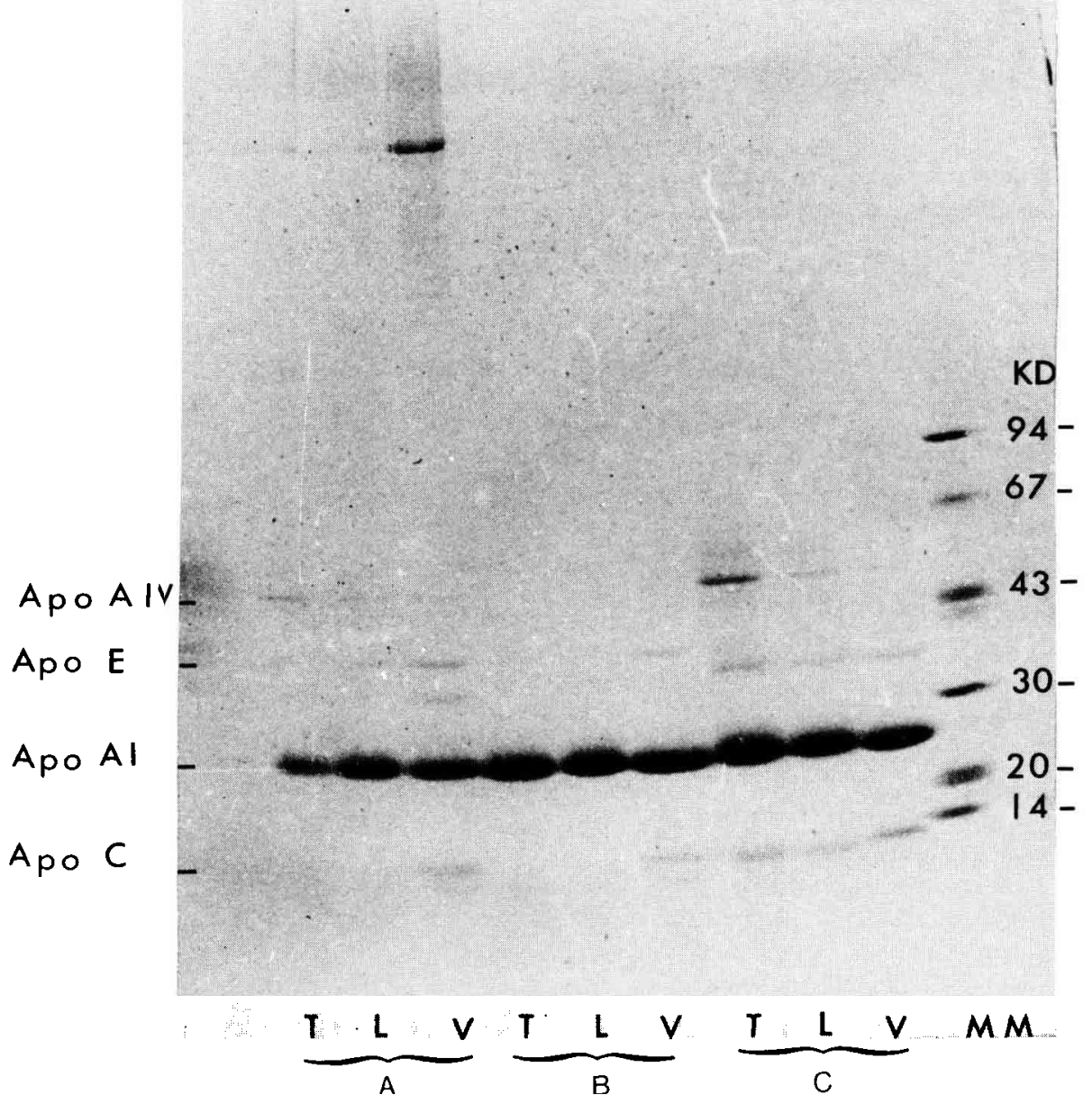

FIG. 1. - Gradient $3-20 \%$ de gel de polyacrylamide en SDS $0,1 \%$. Période : T, tarissement ; L, pleine lactation; $V$, début lactation. $A: 1,006-1,060 ; B: 1,060-1,125 ; C: 1,125-1,21$.

Gloeckler D. H., Ferreri L. F., Flaim E., 1980. Proc. Soc. exp. Biol. Med., 165, 118-122.

Grummer R. R., Davis C. L., 1984. J. Dairy Sci., 67, 2894-2901.

Mazur A., Gueux E., Chilliard Y., Rayssiguier Y., 1986. Reprod. Nutr., Dévelop., 26, 355-356.

Puppione D. L., Raphael B., Mc Carthy R. O., Dimick P. S., 1972. J. Dairy Sci., 55, 265-267.

Raphael B. C., Dimick P. S., Puppione D. L., 1973. J. Dairy Sci., 56, 1025-1032.

Stead D., Welch V. A., 1975. J. Dairy Sci, 58, 112-127. 